\title{
Interplay of physiology, social, familial and behavioural adaptation in the long- term outcome of ARDS
}

\author{
Theodore J Iwashyna, 1,2,3 Timothy S Walsh ${ }^{4,5}$
}

Discharge from a hospital after the intensive care unit (ICU) is challenging. Please do not mistake us-we agree many patients are happy to go home. In fact, most critical care survivors are desperate to leave the hospital, typically after a prolonged frightening hospital stay lasting weeks or longer. Unfortunately, most patients have not returned to their preillness physical status when they go home, and many face new cognitive or psychological disabilities that may just be emerging. Yet, constrained budgets, limited acute hospital beds and wide variation in formal rehabilitation mean many patients enter posthospital survivorship without coordinated support. No matter which health system cares for them, many survivors experience an unplanned rehospitalisation within a few months. ${ }^{1-4}$

Hospital discharge prior to full recovery may be inevitable, as recovery after many illnesses simply takes a really long time (or might never be complete). Data from the Women's Health and Aging Study (all aged $65+$ ) noted $33 \%$ of patients hospitalised for any reason had activity of daily living (ADL) recovery during the 6 months after hospitalisation, and another $14 \%$ in the 6 to 12 -month interval. ${ }^{5}$ (See figure 1) A more acutely ill, younger cohort of critical care patients who required at least 7 days of mechanical ventilation experienced improvement in mean function over the first 6 months, but limited change thereafter. ${ }^{6}$ However, close inspection of these data reveals very wide variation between individuals between 6 and 12 months that are not apparent in population mean estimates. In light of these and related

\footnotetext{
'Department of Internal Medicine, Pulmonary and Critical Care Medicine, Ann Arbor, Michigan, USA ${ }^{2}$ Institute for Social Research, University of Michigan, Ann Arbor, Michigan, USA

${ }^{3}$ Center for Clinical Management Research, VA Ann Arbor Health System, Ann Arbor, Michigan, USA

${ }^{4}$ Centre for Inflammation Research, University of Edinburgh, Edinburgh, UK

${ }^{5}$ Anaesthesia, Critical Care and Pain Medicine, University of Edinburgh, Edinburgh, UK
}

Correspondence to Dr Theodore J Iwashyna, Department of Internal Medicine, University of Michigan, 2800 Plymouth Road, NCRC Building 16, Floor 3, Ann Arbor, MI 48109, USA; tiwashyn@umich. edu

data, our community is rightly expending energy trying to treat this 'post-intensive care syndrome (PICS), ${ }^{7}$ or at least improve the health problems that our patients describe months or years after we discharge them from our ICUs.

As clinicians, we face the challenge of how to interpret the myriad concerns of the recently discharged. We need to decide which concerns will simply resolve on their own, and reassure patients and family about those. Of those that will not resolve on their own, we need to decide which are fixable and which are simply permanent. We need to treat the former and counsel about the latter. To work out how to do this in a practical and cost-effective manner we need research. Clinicians are well aligned with clinical trialists in this area. The clinician seeks a leading indicator of the PICS $^{7}$ to guide their practice. The trialist seeks appropriate outcomes to use in clinical trials. This is where the challenges emerge. We want outcomes that matter to patients, clinicians and healthcare providers, but we do not currently know which are the most appropriate long-term outcomes from critical illness. We therefore tend to use measures that, hopefully, represent key pieces in the mechanistic chain from injury to a less happy life for patients. Many of these earlier pieces are 'surrogate endpoints,' because they may be easier to measure in trials than more complex evaluations.

A surrogate endpoint is something that can be measured early after a treatment that reliably predicts a late, patient-centred outcome. ${ }^{8}$ The crucial characteristic of a surrogate outcome is that it is in the causal pathway of the outcome, so that improvements in the surrogate endpoint mean concurrent or later patient-centred (ie, real and clinically meaningful) outcomes are also improved. Use of CD4 count to predict benefits of HIV therapies is the classic example of an excellent surrogate endpoint. Valid surrogate outcomes in critical care (and often the rest of medicine ${ }^{9}$ ) are frustratingly rare. We can mitigate the harm from wrongly assuming that a surrogate outcome will mean more clinically and economically important outcomes if we understand the relationship among endpoints. As clinicians, a surrogate endpoint is one that we can target during our regular clinical encounters-if we can move the surrogate endpoint, we can have some confidence that we are making patients better.

In Thorax, Chan and colleagues present a valuable secondary analysis of published data relevant to both clinicians and clinical trialists. ${ }^{10}$ In 134 patients from the ARDS Network Long Term Outcome Study (ALTOS) and 99 patients from the Improving Care of Acute Lung Injury Patients (ICAP) cohort study, they examined the inter-relationships over time of a range of outcomes. Their important dependent outcome variables were survival, health-related quality of life (HRQoL) and being alive and at home 6 and 12 months after ICU. These outcomes matter to clinicians, patients and healthcare funders, in part because they are used to calculate quality-adjusted life years which are central to cost-utility analysis.

The elegance of Chan and colleagues' approach was to explore a range of other outcomes that clearly mapped on to different 'levels' of the WHO's International Classification of Functioning, Disability, and Health framework to see if they were associated with these undeniably relevant long-term measures. Past work has shown that health status is poorer among ICU survivors than expected for an age-matched population. This has been shown for measures of function (eg, muscle strength), activity (eg, 6 min walk test) and participation (eg, ADLs). Chan et $a l^{10}$ integrate this large body of work to give us clues to whether these intermediary measures-which are widely interpreted as valid surrogate endpoints-are in reality useful if we want to know about long-term survival and HRQoL after critical illness.

In multivariable analyses, the authors found that physiological measures of structure and function at 6-month follow-up were not associated with survival and HRQoL at 6 and 12 months. Some measures of activity, especially the 6 min walk test, were associated with HRQoL, but not consistently. In contrast, participation measures, which are basically measures of the ability to carry out the instrumental activities of daily living (IADLs), were consistently associated with HRQoL at both time points. Taken together, these findings highlight that differences in muscle strength and activity, and parameters like respiratory function tests that at face value seem 

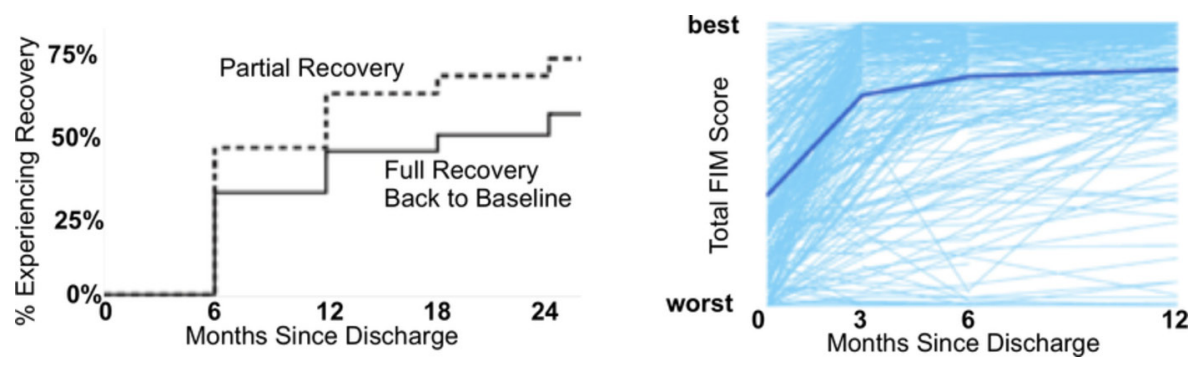

Figure 1 Duration of recovery of function. (Left) Women's Health and Aging Study, all-cause hospitalisation. (Right) Towards RECOVER, $>7$ days intensive care unit ventilation. FIM, functional independence measure.

useful surrogate outcomes, should not be assumed to mirror relevant HRQoL differences.

The data suggest we should concentrate on measuring participation alongside HRQoL by 6 months postdischarge. Knowing this is useful. First, it is actually more difficult (and expensive) to measure structure and function in clinical trials; HRQoL and IADLs can be reliably assessed by telephone or postal questionnaires whereas physical measurements require a time-consuming visit to a clinic (and as a result greater loss to follow-up and less valid data). We could save a lot of research money if we accept these structure and function outcomes are not essential and are not valid surrogate endpoints-at least given the reliability with which they can be measured in actual clinical trial practice. ${ }^{11}$ For clinicians, this means that one should be very tentative about prognosticating based on these physiological measures.

Second, published rehabilitation trials have used a wide range of outcomes; these new data suggest a minimum requirement should be an assessment of IADLs alongside HRQoL in our outcomes if we want patient-focused ways of describing disability. The uncertain validity of structure and function outcomes might also explain why many of our rehabilitation trials have shown no effects on the chosen outcomes. ${ }^{1213}$

There are some notes of caution. All the patients in this analysis had experienced acute respiratory distress syndrome (ARDS) in North America. They may not be representative of more general ICU populations, and in fact they were generally younger and had less comorbidity than 'all comer' ICU groups.

Perhaps more challenging, we do not know-although a survivor and their physician would-how many of these IADL problems at 6 months are new versus how many existed prior to ARDS. Most PICS strategies have focused on prevention or remediation of new deficits; correcting long-standing deficits may present a different challenge. This issue was not the main question asked by Chan and colleagues, but is critical in future research and clinical practice.

In sum, then, Chan and colleagues have taught us some valuable lessons. Although we may love muscle structure and function testing, it does not appear to be a major determinant of 1-year survival or quality of life in their patients with ARDS. This echoes Herridge et al's findings that deficits in pulmonary function are likewise not the major driver of 1-year quality of life in ARDS. ${ }^{14}$ Instead, we may need to orient to the interplay of physiology with social, familial, and behavioural adaptation to understand which of the things we can measure drive the things that matter to patients.

\section{Twitter Follow Theodore J Iwashyna @iwashyna}

Contributors Both authors conceived the manuscript, drafted parts of it and revised it for critical content.

Funding This work was supported by IIR 13-079 from the Department of Veterans Affairs Health Services Research \& Development office.

Disclaimer This does not necessarily represent the view of the US Government or Department of Veterans Affairs.

\section{Competing interests None declared.}

Provenance and peer review Commissioned; externally peer reviewed.

(C) Article author(s) (or their employer(s) unless otherwise stated in the text of the article) 2017. All rights reserved. No commercial use is permitted unless otherwise expressly granted.

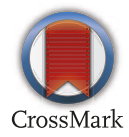

To cite Iwashyna TJ, Walsh TS. Thorax 2017:72:872-873.

Published Online First 1 August 2017

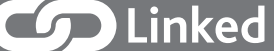

http://dx.doi.org/10.1136/thoraxjnl-2016-209400

Thorax 2017;72:872-873.

doi:10.1136/thoraxjnl-2016-209859

\section{REFERENCES}

1 Prescott HC, Langa KM, Iwashyna TJ. Readmission diagnoses after hospitalization for severe Sepsis and other acute medical conditions. JAMA 2015;313:1055-7.

2 Prescott HC. Variation in Postsepsis Readmission patterns: a Cohort Study of Veterans Affairs beneficiaries. Ann Am Thorac Soc 2017:14:230-7.

3 Lone NI, Gillies MA, Haddow C, et al. Five-Year mortality and hospital costs associated with surviving Intensive Care. Am J Respir Crit Care Med 2016;194:198-208.

4 Liu V, Lei X, Prescott HC, et al. Hospital readmission and healthcare utilization following Sepsis in community settings. J Hosp Med 2014;9:502-7.

5 Boyd CM, Ricks M, Fried LP, et al. Functional decline and recovery of activities of daily living in hospitalized, disabled older women: the women's health and aging study I. J Am Geriatr Soc 2009:57:1757-66.

6 Herridge MS, Chu LM, Matte A, et al. The RECOVER program: disability risk groups and 1-Year outcome after 7 or more days of mechanical ventilation. $A m$ J Respir Crit Care Med 2016;194:831-44.

7 Needham DM, Davidson J, Cohen H, et al. Improving long-term outcomes after discharge from intensive care unit: report from a stakeholders' conference. Crit Care Med 2012;40:502-9.

8 Bucher HC, Guyatt GH, Cook DJ, et al. Users' guides to the medical literature: xix. applying clinical trial results. A. how to use an article measuring the effect of an intervention on surrogate end points. Evidence-Based medicine Working Group. JAMA 1999;282:771-8.

9 Lipska KJ, Krumholz HM. Is hemoglobin A1c the Right Outcome for studies of Diabetes? JAMA 2017:317:1017.

10 Chan KS, Aronson Friedman L, Dinglas VD, et al. Are physical measures related to patient-centred outcomes in ARDS survivors? Thorax 2017;72:884-92.

11 Sjoding MW, Cooke CR, Iwashyna TJ, et al. Acute respiratory distress syndrome measurement error. potential effect on clinical study results. Ann Am Thorac Soc 2016;13:1123-8.

12 Connolly B, Denehy L, Brett $\mathrm{S}$, et al. Exercise rehabilitation following hospital discharge in survivors of critical illness: an integrative review. Crit Care 2012:16:226

13 Connolly B, O'Neill B, Salisbury L, et al. Physical rehabilitation interventions for adult patients during critical illness: an overview of systematic reviews. Thorax 2016:71:881-90.

14 Herridge MS, Cheung AM, Tansey CM, et al. One-year outcomes in survivors of the acute respiratory distress syndrome. N Engl J Med 2003;348:683-93. 